\title{
Interactions between Atropine, Chlorpromazine and Cocaine on Food Reinforced Behavior
}

\author{
MARVIN C. WILSON ${ }^{2}$ AND CHARLES R. SCHUSTER ${ }^{3}$ \\ Pharmacology Department, Medical School, University of Michigan, Ann Arbor, MI 48105
}

(Received 23 August 1974)

\begin{abstract}
WILSON, M. C. AND C. R. SCHUSTER. Interactions between atropine, chlorpromazine and cocaine on food reinforced behavior. PHARMAC. BIOCHEM. BEHAV. 3(3) 363-375, 1975. - Previous investigations have demonstrated the ability of atropine and chlorpromazine pretreatment to increase intravenous cocaine self-administration in rhesus monkeys. These results were interpreted as resulting from either an antagonism of cocaine's reinforcing effect or an effect of cocaine which may interfere with its self-administration. The generality of the postulated drug antagonism was tested in monkeys exhibiting stable FR10 food-reinforced behavior. Intramuscular pretreatment with $0.25-4.0 \mathrm{mg} / \mathrm{kg}$ of chlorpromazine significantly depressed this behavior as did pretreatment with $0.25-2.0 \mathrm{mg} / \mathrm{kg}$ of atropine. These same dosages had been shown to increase cocaine self-administration. Fifteen-minute influsions of cocaine $(1.0-10$ $\mathrm{mg} / \mathrm{kg}$ ), which began $15 \mathrm{~min}$ after session onset, also resulted in a depression of food reinforced behavior. When chlorpromazine pretreatment and cocaine infusion were combined, the decrement in food reinforced responding was lessened. However, when cocaine infusion and atropine pretreatment occurred concurrently, the depression in responding was increased. These data indicate that chlorpromazine may nonspecifically antagonize the behavioral effects of cocaine; whereas, atropine may enhance these effects.
\end{abstract}

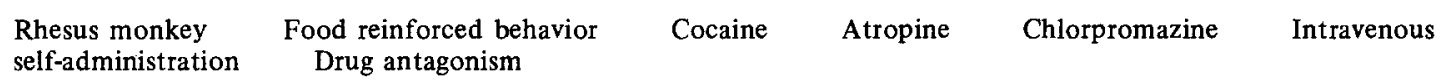

PREVIOUS investigations have demonstrated that, when allowed limited daily access to psychomotor stimulants, rhesus monkeys demonstrate a marked stability in intravenous self-administration behavior $[5,11,15]$. Furthermore, reductions in unit dosage, the amount of psychomotor stimulant administered per infusion, within a range of reinforcing dosages, increases the frequency of selfadministration behavior. As a result, monkeys daily selfadminister approximately the same total amount of psychomotor stimulants e.g. cocaine, phenmetrazine, methylphenidate, and pipradrol, regardless of unit dosage.

If animals emitting stable self-administration behavior are treated intramuscularly with certain dosages of phenothiazines i.e., chlorpromazine and trifluoperazine, or with atropine prior to the onset of the daily session, the amount of cocaine self-administered and the frequency of self-administration behavior increase $[12,13,14]$. Since mechanisms underlying the generation of this stable selfadministration behavior are obscure, it is difficult to postulate the mechanism of drug induced increases in self-administration behavior. Since a reduction in unit dosage increases the frequency of self-administration behavior, one possible explanation would be that these agents are par- tially antagonizing the reinforcing action of these stimulants. Alternatively these agents may be antagonizing an aversive or behavioral disrupting action e.g.; a competing behavior such as intense stereotypy, which may normally function in limiting the amount of a psychomotor stimulant which is self-administered. Furthermore, if these agents antagonize an aversive action of psychomotor stimulants, the reinforcing efficacy of these agents may be increased.

A study of the interaction in monkeys between cocaine and atropine, and between chlorpromazine and cocaine on some other operant behavior e.g. food reinforced behavior, may assist in ascertaining the specificity as well as the mechanisms responsible for the effects of these two agents on cocaine self-administration behavior.

\section{METHOD}

\section{Animals and Apparatus}

The animals for this study were three adult male experimentally naive rhesus monkeys weighing between 4.0 and $4.6 \mathrm{~kg}$. Animals were housed individually in completely enclosed experimental cubicles. The top, sides and upper half of the back wall were constructed of nontransparent

\footnotetext{
${ }^{1}$ This research was supported by NIMH Grants 12084,18245 and 11052 .

${ }^{2}$ Presently located in the Department of Pharmacology, School of Pharmacy, University of Mississippi, University, Mississippi 38677.

${ }^{3}$ Presently located in the Departments of Pharmacology and Psychiatry, University of Chicago, 950 East 59th Street, Chicago, Illinois 60637.
} 
white plastic. The floor of the cubicle consisted of $2.54 \mathrm{~cm}$ glavanized wire mesh. An aluminum door served as the front of the cage. The bottom half of the back wall consisted of a stainless steel panel. A Foringer food pellet dispenser was mounted on the outside of the front door. This was connected via metal tubing which passed through a $2.54 \mathrm{~cm}$ hole in the door to a food cup mounted on the inside of the door. An exhaust fan was mounted on the outside in the middle of the cubicle ceiling. This fan was constantly operated to provide a constant temperature inside the cubicle and also to mask some of the noise occurring in the laboratory since the cubicles were not sound-proofed. Located on the stainless steel panel on the back wall of the cubicle were a red stimulus light (4 watts), a pressure sensitive water spout to provide ad lib water, and a response lever. A food container accessible to both the inside and outside of the cubicle was located in a back corner of the cubicle at floor level.

Animals were restrained in the cubicle by a stainless steel arm and harness [4]. The restraining arm was attached to the back of the cubicle. This method of restraint allowed relatively free movement of the animal throughout the cubicle and also protected an intravenous catheter system. An indwelling jugular catheter of siliconized rubber (I.D. = $0.61 \mathrm{~mm}$, O.D. $=1.82 \mathrm{~mm}$ ) was attached to a needle connector located on the harness; a second piece of tubing, originating at this connector, traversed the lumen of the restraining arm and was attached to a Harvard infusion pump. The infusion pump was mounted on a stainless steel metal shelf attached to the outside of the rear wall of the cubicle. Cables connected the apparatus associated with the cubicles to electromechanical switching and recording apparatus located in an adjacent room.

\section{Procedure}

Following placement in the cubicles which then became the animals' home cages, the animals were fed 15 Purina Monkey Chows twice daily at 8:30 a.m. and 4:30 p.m. This food was normally consumed within $30 \mathrm{~min}$. Following 7 days of adaptation to this food access schedule, these animals were fed only once daily, at 4:30 p.m. Under these conditions of food deprivation, animals were conditioned to lever press for 0.7 gram Dietrich and Gambrell food pellets. Initially each lever press response exceeding $100 \mathrm{~g}$ of force occurring in the presence of stimulus light illumination was reinforced with presentation of a single pellet. The stimulus light was illuminated daily from 11:00 a.m. to 1:00 p.m. Subsequently, the requirements for reinforcement were increased to 10 responses (FR10). Following each pellet presentation a 1 min time-out ensued during which the stimulus light was turned off and lever press responses, although recorded, had no consequence. Behavior was recorded on Gerbrands cumulative recorders which were inoperative during the time-out period; however, the session clock continued to operate. These contingencies limited the maximum number of pellets which could be received in a session to less than 120 .

After each animal's performance on this reinforcement schedule had stabilized, a chronic indwelling catheter was implanted in an internal jugular vein. Anesthesia was accomplished by slowly administering $30 \mathrm{mg} / \mathrm{kg}$ of pentobarbital sodium intravenously. Following surgery 600,000 units of penicillin, i.e., 300,000 units of benzathine penicillin $G$ and 300,000 units of procaine penicillin $G$ suspension
(Bicillin C-R by Wyeth) was administered intramuscularly as a prophylactic anti-infective measure. Beginning with the following experimental session, each presentation of a food pellet was accompanied by the intravenous administration of sterile physiological saline $(0.2 \mathrm{cc} / \mathrm{kg}$ of body weight). This was done to retard clot formation in the catheter. Initially responding during the sessions decreased but returned to control values within 7 sessions.

The effects of the intramuscular administration of atropine and chlorpromazine on this baseline behavior were determined in each of the 3 animals. Pretreatment dosgages of $0.25,1.0$ and $2.0 \mathrm{mg} / \mathrm{kg}$ of atropine sulfate, and 0.125 , $0.25,0.5,1.0,2.0$ and $4.0 \mathrm{mg} / \mathrm{kg}$ of chlorpromazine hydrochloride (Thorazine by Smith, Kline and French) were administered $5 \mathrm{~min}$ prior to session onset. The drugs were dissolved in sterile physiological saline and all solutions were prepared on the day on which they were administered. The injection volume was held constant at $1.0 \mathrm{ml}$. At least 3 control sessions intervened between drug pretreatment sessions to permit the return of behavioral stability prior to the next drug administration. Five control sessions in which animals were pretreated intramuscularly with sterile physiological saline were conducted in each animal during the period of testing each drug. Each dosage of each drug was tested once in each animal in randomized fashion. Treatment with one substance was completed before initiating treatment with the other drug.

Following the determination of the effects of these two substances on this behavior, the effects of cocaine were ascertained. Cocaine hydrochloride in dosages of $0.5,1.25$, $2.5,5.0$ and $10 \mathrm{mg} / \mathrm{kg}$ were tested in each of the 3 animals in a randomized fashion. These dosages were administered via slow intravenous infusion via the jugular catheter. The infusion of cocaine hydrochloride in sterile physiological saline occurred over $15 \mathrm{~min}$ and the infusion volume was kept constant at $20 \mathrm{ml}$. The infusion began $15 \mathrm{~min}$ after the onset of the session. This time was chosen to approximate the time of the onset of drug action following the intramuscular treatment with atropine and chlorpromazine. Cocaine solutions were prepared on the day in which they were administered. Three days elapsed between drug treatment sessions. Five control sessions i.e., $15 \mathrm{~min}$ infusions of $20 \mathrm{ml}$ of sterile physiological saline, were conducted in each animal. During cocaine and saline infusions, completion of each tenth response resulted in pellet presentation.

Following the determination of the acute effects of each of the dosages of atropine, chlorpromazine and cocaine on food reinforced behavior in each animal, the effects of administering combinations of these drugs were ascertained in 2 of the 3 animals. Drugs were administered at the same time and by the same route as previously. Each combination was administered to each animal. Appropriate salinesaline and drug-saline controls were conducted.

\section{RESULTS}

The effects of chlorpromazine on fixed-ratio food reinforced behavior are illustrated in Fig. 1. Pretreatment with $0.125 \mathrm{mg} / \mathrm{kg}$ did not effect the frequency of food reinforced responding. Increasing the pretreatment dosage to $0.25 \mathrm{mg} / \mathrm{kg}$ resulted in a significant $(p<0.05)$, decrease when compared to the effect of saline, in the number of food reinforcements received during the session as determined by the Student's $t$. Further increases in the pretreatment dosage of chlorpromazine i.e., $0.5-4.0 \mathrm{mg} / \mathrm{kg}$, re- 


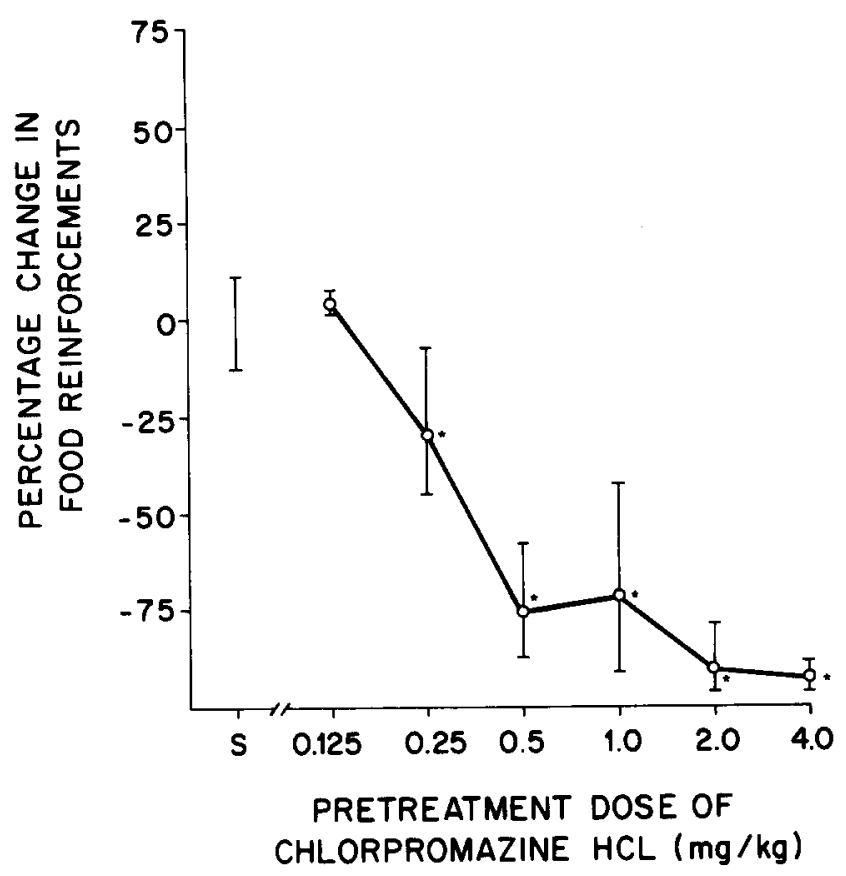

FIG. 1. Mean percentage change in the number of food pellets received during the entire session as a function of the pretreatment dosage of chlorpromazine. Each point represents the mean of 3 animals and the vertical lines indicate the range. The brackets at the left represent the range of effect produced by saline pretreatment in 15 sessions ( 5 conducted per animal). An asterisk $\left({ }^{*}\right)$ denotes those points statistically different from saline as determined by use of the Student's $t$ Test for significance $(p<0.05)$.

sulted in a large decrement in the number of food reinforcements received. Associated with these decrements in food reinforced behavior was gross observational evidence of nonspecific CNS depression. This was demonstrated by a reduction in locomotor activity, and in responsiveness to novel stimuli. Catalepsy was evident at dosages of 2.0 and $4.0 \mathrm{mg} / \mathrm{kg}$.

Figure 2 illustrates the cumulative recorder records of those sessions following chlorpromazine pretreatment in one animal, which was typical of the remaining animals. The onset of chlorpromazine's effect on this behavior was dose related. The effects of the 0.25 and $0.5 \mathrm{mg} / \mathrm{kg}$ dosages were apparent within fifteen minutes after onset of the session; however, the onset of action of the $1.0,2.0$ and $4.0 \mathrm{mg} / \mathrm{kg}$ dosages was well within $8 \mathrm{~min}$ of session onset. These larger dosages of chlorpromazine abruptly terminated food reinforced behavior. Furthermore, the duration of the decrement in food reinforced behavior was dose dependent. The effect of $0.25 \mathrm{mg} / \mathrm{kg}$ lasted about $1 \mathrm{hr}$; whereas, 0.5 and $1.0 \mathrm{mg} / \mathrm{kg}$ depressed this behavior for $90 \mathrm{~min}$. Pretreatment with 2.0 and $4.0 \mathrm{mg} / \mathrm{kg}$ completely suppressed this behavior from 5 min post session onset until the session terminated.

The effects of atropine pretreatment on food reinforced behavior are demonstrated in Fig. 3. Atropine sulfate in the dosage range of $0.25-2.0 \mathrm{mg} / \mathrm{kg}$ produced a dose related depression in this behavior. The effect of each of these dosages was statistically significant from saline. There were no observable signs of central depression or stimulation produced by these dosages of atropine; however, mydriasis was prominent. The cumulative response records of the atropine pretreatment sessions conducted in one subject are illustrated in Fig. 4. The onset of the effect of atropine on this behavior was in all cases within 5 min after onset of the session. The magnitude and the duration of atropine's action were dose dependent. The reduction in responding lasted for the entire session with the $0.5,1.0$ and $2.0 \mathrm{mg} / \mathrm{kg}$ dosages, although there was partial behavioral recovery, lasting only a few minutes, midway through the session following the $0.5 \mathrm{mg} / \mathrm{kg}$ dose.

The effect of the intravenous administration of cocaine on food reinforced behavior is illustrated in Fig. 5. Cocaine in dosages of $0.25,0.5$ and $1.0 \mathrm{mg} / \mathrm{kg}$ did not significantly alter the number of food reinforcements obtained during the entire session. Infusions of $2.5,5.0$ and $10 \mathrm{mg} / \mathrm{kg}$ did significantly decrease this behavior when compared to saline infusions. Lever press behavior was respectively suppressed by $50 \%, 70 \%$ and $71 \%$. These latter two values were essentially a lower limit since food reinforced responding stopped abruptly at the onset of the infusion and was not reinstated during the remainder of the session. Approximately $25 \%$ of the food pellets obtained during saline infusion sessions occurred prior to infusion onset; therefore, it would have been highly improble to obtain a decrement larger than $75 \%$.

The cumulative recorder records of those sessions obtained from one animal in which various dosages of cocaine were infused, as well as the record of an appropriate saline infusion control session, are illustrated in Fig. 6. Food reinforced behavior was not altered by the saline infusion. Fifteen minute cocaine infusions in dosages of $0.5-5.0$ $\mathrm{mg} / \mathrm{kg}$ produced a disruption in behavior soon after the infusion began. The magnitude and duration of the behavioral disruption were dose dependent. In all cases responding was greatly reduced during the duration of the infusion. Furthermore, infusion of $5.0 \mathrm{mg} / \mathrm{kg}$ completely suppressed food-reinforced behavior for the remainder of the session. Mydriasis, piloerection, salivation, increased reactivity to novel stimuli and increased grooming behavior were evident following infusions of 2.5 and $5.0 \mathrm{mg} / \mathrm{kg}$. Although not illustrated in this figure due to a programming malfunction, the effect of an infusion of $10 \mathrm{mg} / \mathrm{kg}$ of cocaine was very similar to that produced by the effect of $5.0 \mathrm{mg} / \mathrm{kg}$ except the onset of response suppression was more rapid with the higher dose.

Therefore, atropine pretreatment, chlorpromazine pretreatment and the intravenous administration of cocaine all produced a dose related suppression of food reinforced behavior. The effects of combining intramuscular chlorpromazine treatment and intravenous cocaine treatment on FR-10 food reinforced behavior in both animals are illustrated in Fig. 7. The points represent the number of food reinforcements obtained during the last 1.05 minutes, of the two hour session. In both animals a combination of chlorpromazine pretreatment, 0.25 and $0.5 \mathrm{mg} / \mathrm{kg}$ and saline infusion produced a dose related decrease in food reinforced behavior when compared to sessions in which the same animals received a combination of saline pretreatment and saline infusion. Furthermore, in one of the two animals (335) a combination of saline pretreatment and cocaine infusions, 0.25 and $0.5 \mathrm{mg} / \mathrm{kg}$, produced a dose related decrease in food reinforced behavior. In both 335 and 338 , infusions of 0.25 and $0.5 \mathrm{mg} / \mathrm{kg}$ of cocaine antagonized, in a dose related manner, the effect of pretreatment 

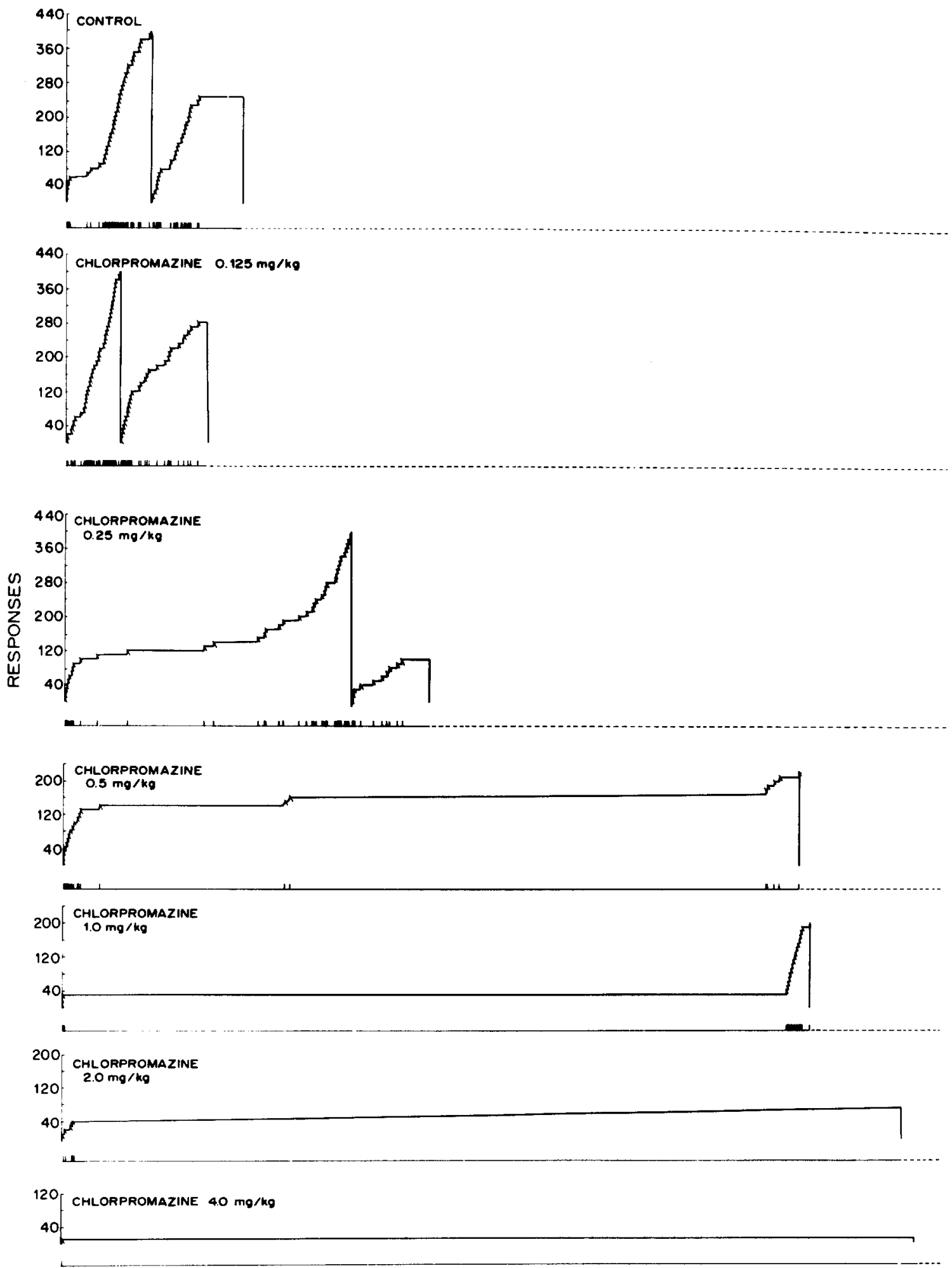

FIG. 2. Cumulative response records which illustrate the effects of chlorpromazine on food reinforced behavior in 1 animal. Each vertical mark on the horizontal line represents presentation of 1 food pellet i.e. 10 responses were emitted between successive vertical lines. The end of the $2 \mathrm{hr}$ session is indicated by the change in character of the horizontal line from a solid to a broken form. The response pen reset after each 400 responses and at the end of the session. The recorder was stopped during the $1 \mathrm{~min}$ timeout following each reinforcement, but the session timer continued to operate. Therefore, the length of the solid horizontal line is inversely proportional to the number of reinforcements received i.e. frequency of responding, during the session. 


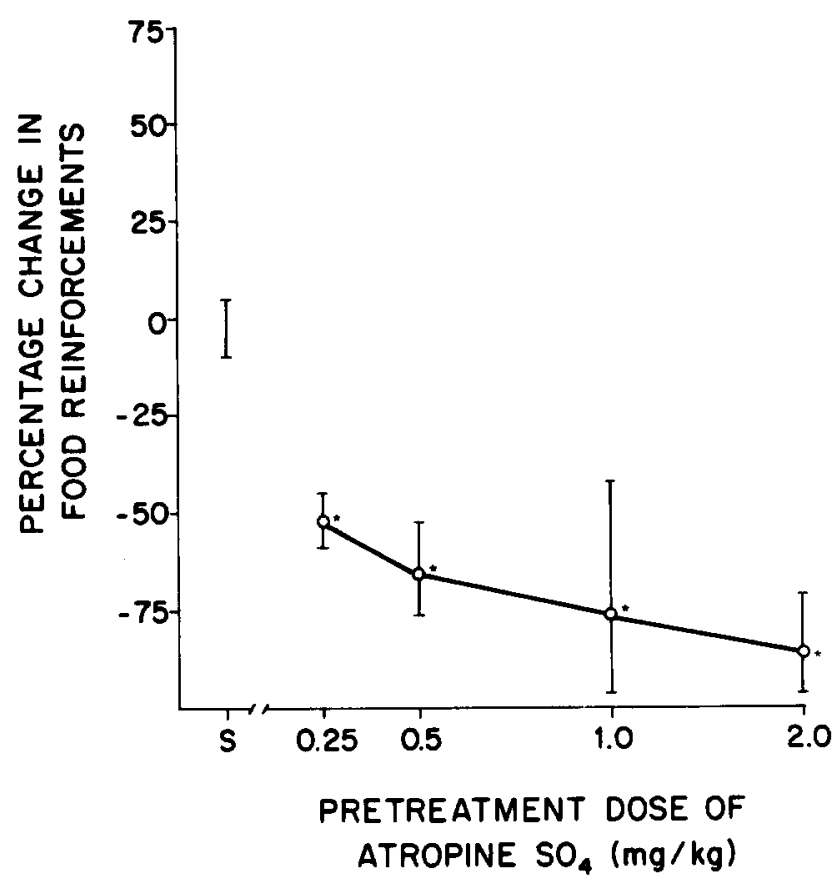

FIG. 3. Mean percentage change in the number of food pellets received during the entire session as a function of the pretreatment dosage of atropine. Each point represents the mean of 3 animals and the vertical lines indicate the range. The brackets at the left represent the range of effect produced by saline pretreatment in fifteen sessions ( 5 conducted per animal).

with chlorpromazine, 0.25 and $0.5 \mathrm{mg} / \mathrm{kg}$. In 338 , cocaine at both dosages completely blocked the effect of 0.25 $\mathrm{mg} / \mathrm{kg}$ of chlorpromazine. In $335,0.25 \mathrm{mg} / \mathrm{kg}$ of chlorpromazine blocked the depressant effect of $0.5 \mathrm{mg} / \mathrm{kg}$ of cocaine on this behavior.

The cumulative records of those sessions in which Animal 335 was treated individually with saline, cocaine, or chlorpromazine and with combinations of these substances are illustrated in Fig. 8. The initial record is from a session in which this animal received both an intramuscular pretreatment, as well as an infusion, of saline. The response rate was very stable throughout the entire session. Pretreatment with $0.5 \mathrm{mg} / \mathrm{kg}$ of chlorpromazine greatly suppressed food reinforced behavior after approximately $15 \mathrm{~min}$ of the session had elapsed. This response suppression was present throughout the remainder of the session. As indicated in Fig. 7, in fusions of 0.25 and $0.5 \mathrm{mg} / \mathrm{kg}$ of cocaine antagonized the depressant action of chlorpromazine. The duration of the chlorpromazine induced suppression of responding was reduced in a dose dependent manner by the cocaine infusions. Responding during the cocaine infusions seldom occurred. This was not surprising since when this animal received saline pretreatment plus cocaine infusions responding did not occur during the infusions.

The effects of atropine pretreatment and cocaine infusions individually and in combination on food reinforced responding are illustrated in Fig. 9. Atropine pretreatment in dosages of 0.25 and $0.5 \mathrm{mg} / \mathrm{kg}$ greatly reduced food reinforced responding in a dose related manner during the last $105 \mathrm{~min}$ of the $2 \mathrm{hr}$ session in both animals. Cocaine infusions of $0.25-5.0 \mathrm{mg} / \mathrm{kg}$ also reduced responding in a dose related manner in 335 ; however, only doses of 2.5 and $5.0 \mathrm{mg} / \mathrm{kg}$ were effective in reducing responding in 338 . Unlike the interaction between cocaine and chlorpromazine, combinations of cocaine and atropine always resulted in either no change or in a greater decrement in responding than that which occurred following treatment with atropine alone. There was some indication in subject 338 that pretreatment with $0.25 \mathrm{mg} / \mathrm{kg}$ of atropine may to some extent attenuate the response suppression resulting from an infusion of $5.0 \mathrm{mg} / \mathrm{kg}$ of cocaine.

Figure 10 illustrates the cumulative recorder records obtained from sessions conducted in one animal in which cocaine and/or atropine were administered. The records from the other animal were quite similar in nature. Pretreatment with atropine and a saline infusion produced a decrement in the rate of food reinforced responding, which lasted throughout the entire session, when compared to the record obtained from a control session in which pretreatment with saline was combined with a saline infusion. The bottom two records indicate that saline pretreatment, combined with a cocaine infusion $(0.25,0.5 \mathrm{mg} / \mathrm{kg})$ resulted in cessation of responding during the infusion. Responding was quickly reinstated at the control rate following termination of the $0.25 \mathrm{mg} / \mathrm{kg}$ infusion. Cessation of responding lasted for approximately $10 \mathrm{~min}$ following termination of $0.5 \mathrm{mg} / \mathrm{kg}$ infusion. When atropine pretreatment and cocaine infusions were combined at all dosage values tested, the resulting decrement in responding was greater and longer in duration, with one exception, than that seen following treatment with either of the agents alone. The effects of pretreatment with $0.25 \mathrm{mg} / \mathrm{kg}$ of atropine produced qualitatively but not quantitatively similar results on all occasions. The response rate seen in Records $2-6$, prior to the onset of either saline or cocaine infusions, was depressed in each case when compared to the first record. However, on some occasions this dosage of atropine was more effective in depressing this behavior than on others.

\section{DISCUSSION}

The decrement in food reinforced behavior produced by chlorpromazine may have been the result of a specific anorexic effect of this agent but more likely the result of a nonspecific neuroleptic action. Certainly the gross observational appearance of the subjects after pretreatment with dosages which significantly depressed lever press behavior tend to support such a conclusion. Evidence of central depression was present only on those occasions during which food intake was significantly depressed.

If one can assume that this effect of chlorpromazine on food reinforced responding is non-selective, it is interesting to note that dosages which significantly depressed this behavior, facilitate cocaine self-administration [12]. One could then speculate that the self-administered cocaine antagonized this depressant action of the chlorpromazine which then permitted further cocaine self-administration. This then would be indicative of the existence of a mutual antagonism between cocaine and chlorpromazine.

Decreases in food reinforced responding following treatment with atropine have been previously demonstrated [10]. The decrease in food intake which occurred was interpreted as being the result of atropine's potent de- 
No. 338 FR-10 (FOOD PELLETS)
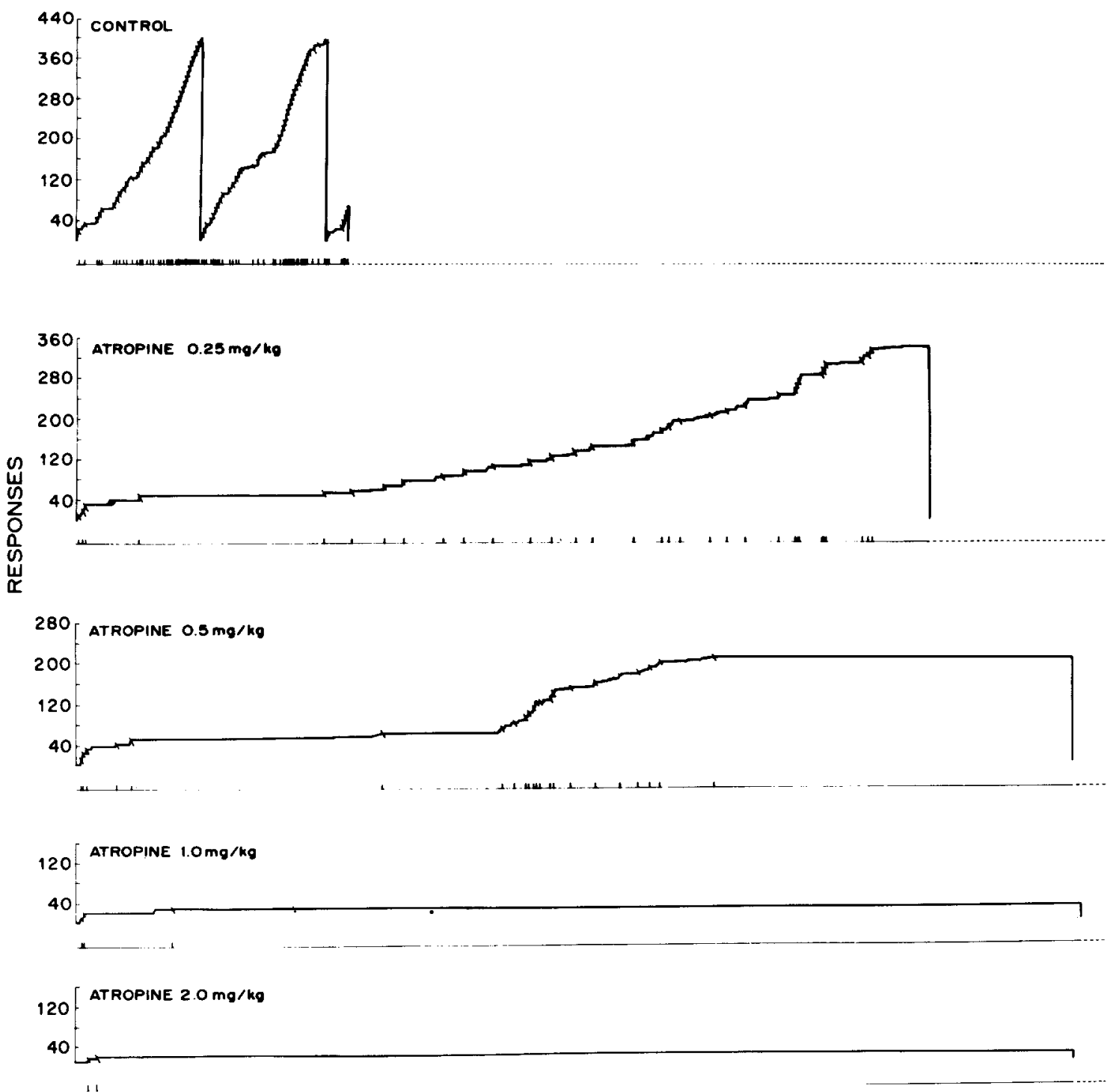

FIG. 4. Cumulative response records which illustrate the effects of atropine on food reinforced behavior in 1 animal. Each vertical mark on the horizontal line represents presentation of one food pellet i.e. 10 responses were emitted between successive vertical lines. 


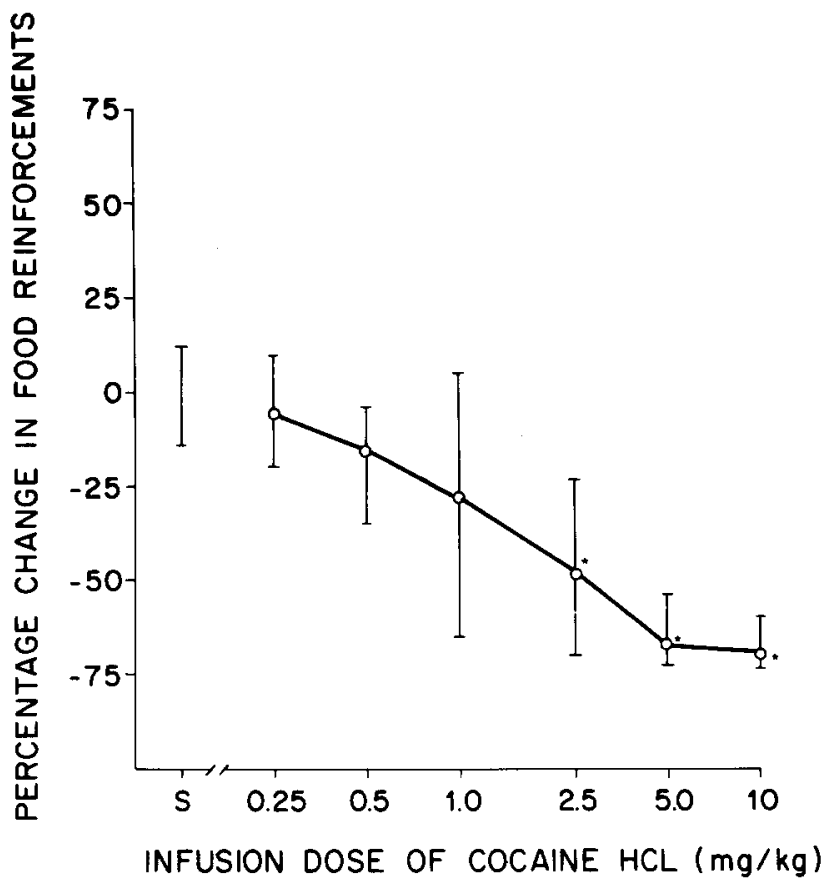

FIG. 5. Mean percentage change in the number of food pellets received during the entire session as a function of the treatment dosage of cocaine. Each point represents the mean of 3 animals and the vertical lines indicate the range. The brackets at the left represent the range of effect produced by saline infusions in $\mathbf{1 5}$ sessions ( 5 conducted per animal)

pressing action on salivation since these investigators obtained similar results after treatment with methylatropine. In the present study water was freely available during the sessions so the effect on salivation i.e., dry mouth, could have been overcome by the animal and food easily consumed. Furthermore, agents capable of increasing salivation eg., cocaine, should be able to partly reverse this effect on food reinforced responding if it is entirely the result of a dry mouth. The depression of food reinforced behavior exerted by atropine in the present study was not the result of any nonselective behavioral disruption. In fact the animals appeared to demonstrate a facilitation of unconditioned behavior e.g. locomotor activity, hyperactivity, during sessions following pretreatment with 1.0 and 2.0 $\mathrm{mg} / \mathrm{kg}$. Furthermore, pretreatment with $0.5-2.0 \mathrm{mg} / \mathrm{kg}$ of atropine has been shown to facilitate intravenous cocaine self-administration in this same species [14]. Therefore, it may be hypothesized that the decrement in food reinforced responding produced by atropine is not simply the result of its antisecretory effect on the salivary glands, but also may include a central component.

The behavioral disrupting effect of intravenously administered cocaine on fixed ratio food reinforced responding has been demonstrated in the rat [7]. The length of the disruptions were dose dependent; therefore, these results are in agreement with the present data. The pausing in food reinforced behavior observed in the rat following each administration was similar in duration to that which occurred during self-administration sessions following each intravenous self-administration of the same dosage.
The ability of cocaine, self-administered intravenously by rhesus monkeys, to disrupt food reinforced behavior has been previously reported [1]. These investigators utilized a fixed interval 9 minute schedule of reinforcement with a limited hold of three minutes. A fifteen minute timeout followed presentation of each reinforcer. Three successive cocaine infusions, which were separated by a 24-27 minute interval, were followed in 24 minutes by access to a single food reinforcement. During a daily session subjects had the opportunity of self-administering a total of 30 infusions of cocaine and 10 food pellets. Infusions of 0.4 and $0.8 \mathrm{mg} / \mathrm{kg}$ of cocaine resulted in a suppression of food reinforced responding. Whereas lower unit dosages $0.025-0.2 \mathrm{mg} / \mathrm{kg}$ did not alter this behavior. In the present study a significant decrease in food reinforced was not observed until the infusion dosage of cocaine had been increased to $2.5 \mathrm{mg} / \mathrm{kg}$, although in some animals a large decrement in responding was achieved with a $1.0 \mathrm{mg} / \mathrm{kg}$ dose. The apparent greater sensitivity of the animals in the Balster and Schuster study to the rate suppressing effect of cocaine of food reinforced behavior may have resulted from the different reinforcement schedules utilized in these two studies. A fixed interval schedule of food reinforcement is usually considered to be more sensitive to drug effects than a fixed ratio schedule of reinforcement, which was used in the present study. These studies then demonstrate that the intravenous administration of cocaine may reduce food reinforced responding via either a selective anorexic effect or by a nonselective behavioral disrupting action.

It is interesting that the infusion of cocaine could antag- 
370

WILSON AND SCHUSTER

No. $216 \uparrow$ FR -10 (FOOD PELLETS)
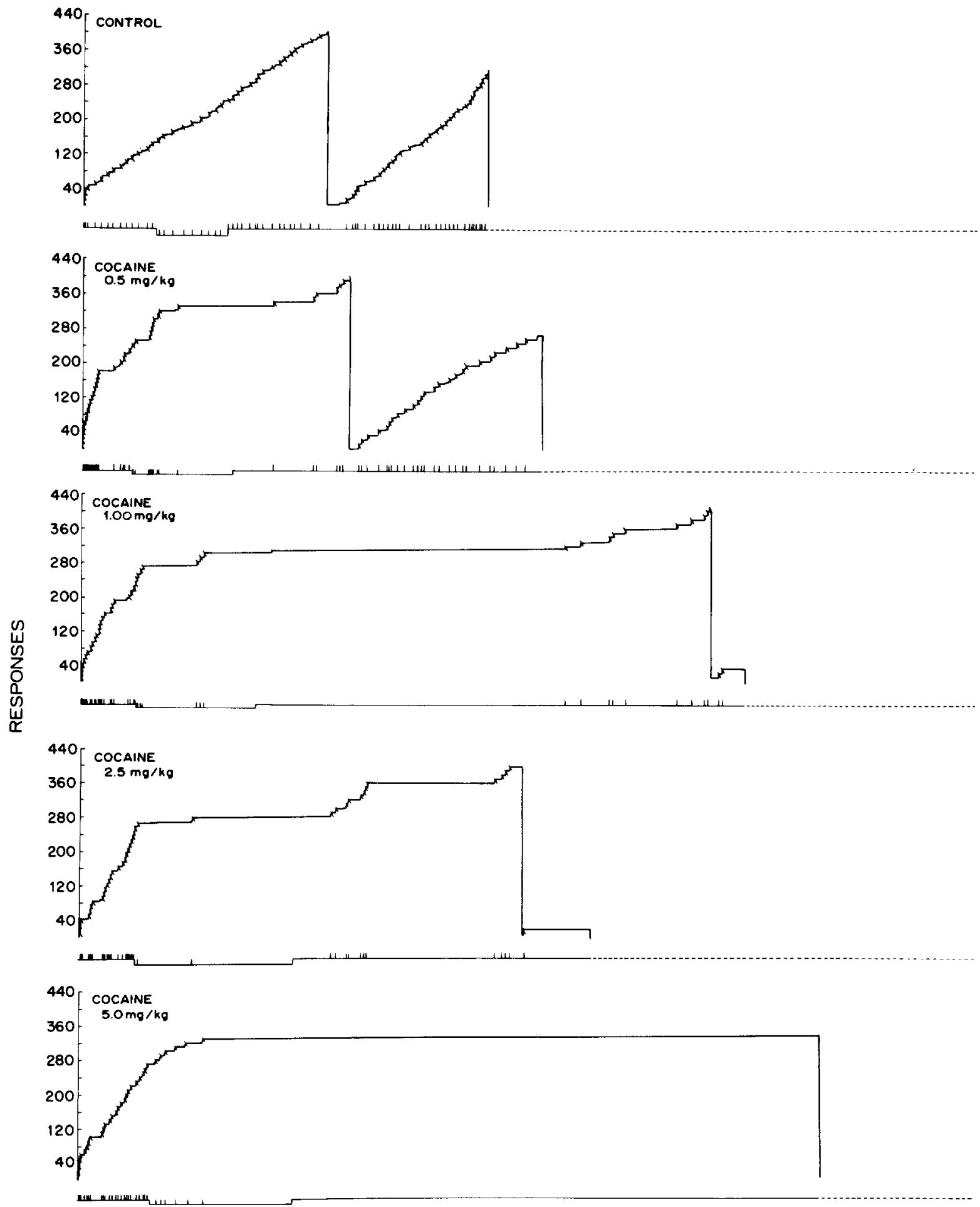

FIG. 6. Cumulative response records which illustrate the effects of cocaine infusions on food reinforced behavior in 1 animal. Each vertical mark of the horizontal line represents presentation of 1 food pellet i.e. 10 responses were emitted between successive vertical lines. The end of the $2 \mathrm{hr}$ session is indicated by the change in character of the horizontal line from a solid to a broken form. The negative displacement of the horizontal line indicates the onset of the saline or cocaine infusion. The length of the infusion is indicated by the horizontal length of this downward deflection. The actual length of the infusion was kept constant but since the recorder was stopped during the $1 \mathrm{~min}$ timeout following each reinforcement, the length of the offset may vary depending on whether responding occurred during the infusion. The length of the solid horizontal line is inversely proportional to the number of reinforcements received i.e. frequency of responding, during the session since the recorder stopped after each reinforcement. 


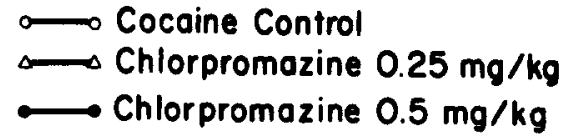

No. 335
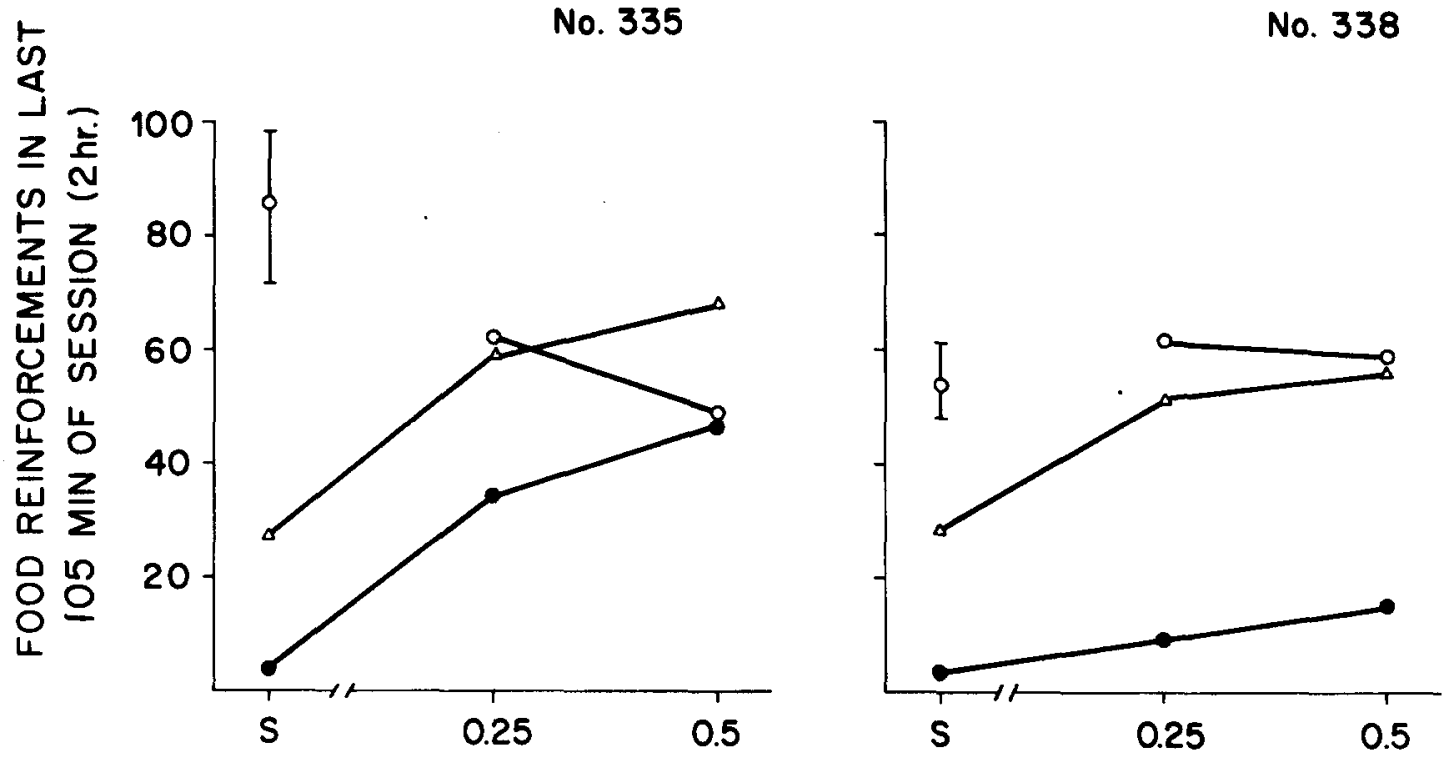

INFUSION DOSE OF COCAINE HCL $(\mathrm{mg} / \mathrm{kg})$

FIG. 7. Number of food reinforcements obtained during the last hour and $45 \mathrm{~min}$ of the $2 \mathrm{hr}$ session as a function of the pretreatment dosage of chlorpromazine and the infusion dosage of cocaine, individually and in combination. The brackets and superimposed symbol indicate the range and mean obtained from 5 control sessions i.e. animal received both a saline pretreatment and a saline infusion. The points above the letter " $\mathrm{S}$ " indicate the effects seen with chlorpromazine pretreatment alone.

onize the depressant effect of chlorpromazine on food reinforced behavior and that in one of the animals, chlorpromazine pretreatment reduced the effect of cocaine infusions on this behavior. This might indicate that (1) cocaine may reduce the nonselective behavioral suppressant or neuroleptic effects of chlorpromazine and (2) that chlorpromazine can antagonize either the anorexic or behavioral disrupting effects of cocaine which reduced food reinforced responding. One might speculate that this latter mechanism is responsible for the ability of the phenothiazines to enhance cocaine self-administration behavior [12]. Other investigators have demonstrated an antagonism between chlorpromazine and another psychomotor stimulant, d-amphetamine, on a food reinforced operant in rats $[2,6]$. Treatment with a dosage of chlorpromazine which did not depress food reinforced behavior, antagonized the decrease in this behavior produced by d-amphetamine. Therefore the nonspecific behavioral suppression exerted by the phenothiazines may not be required to antagonize the behavioral effects of psychomotor stimulants. This, however, is not evidenced by data from the present study although previous data from this laboratory has demonstrated that a dosage of chlorpromazine which does not decrease food reinforced behavior $(0.125 \mathrm{mg} / \mathrm{kg})$ does enhance cocaine self-administration [12].
Quite unlike the results with chlorpromazine, cocaine infusions of dosages which either had no effect on food reinforced responding or which decreased responding, enhanced the decrement in food reinforced responding produced by atropine. This is consistent with most of the behavioral literature concerning the combined actions of atropine and psychomotor stimulants.

Methamphetamine induced increases in intracranial selfstimulation in rats are enchanced by atropine [9]. Atropine has also been demonstrated to potentiate the effects of d-amphetamine and of cocaine on continuous avoidance behavior. Neither the dose of atropine, cocaine, nor damphetamine used in the combination treatments facilitated avoidance behavior when administered individually $[3,8]$. Atropine also enhances cocaine induced hyperactivity [8]. Therefore it may have been expected that atropine and cocaine may enhance each other's effects on food reinforced behavior. Therefore the present data are in agreement with that obtained in other behavioral paradigms.

Therefore, since atropine pretreatment in the present study, in dosages which have been demonstrated to facilitate cocaine self-administration behavior, enhanced rather than antagonized the effect of cocaine on food reinforced behavior, the effect on self-administration is not the result 
372

WILSON AND SCHUSTER

No. 335 FR-10 (FOOD PELLETS)
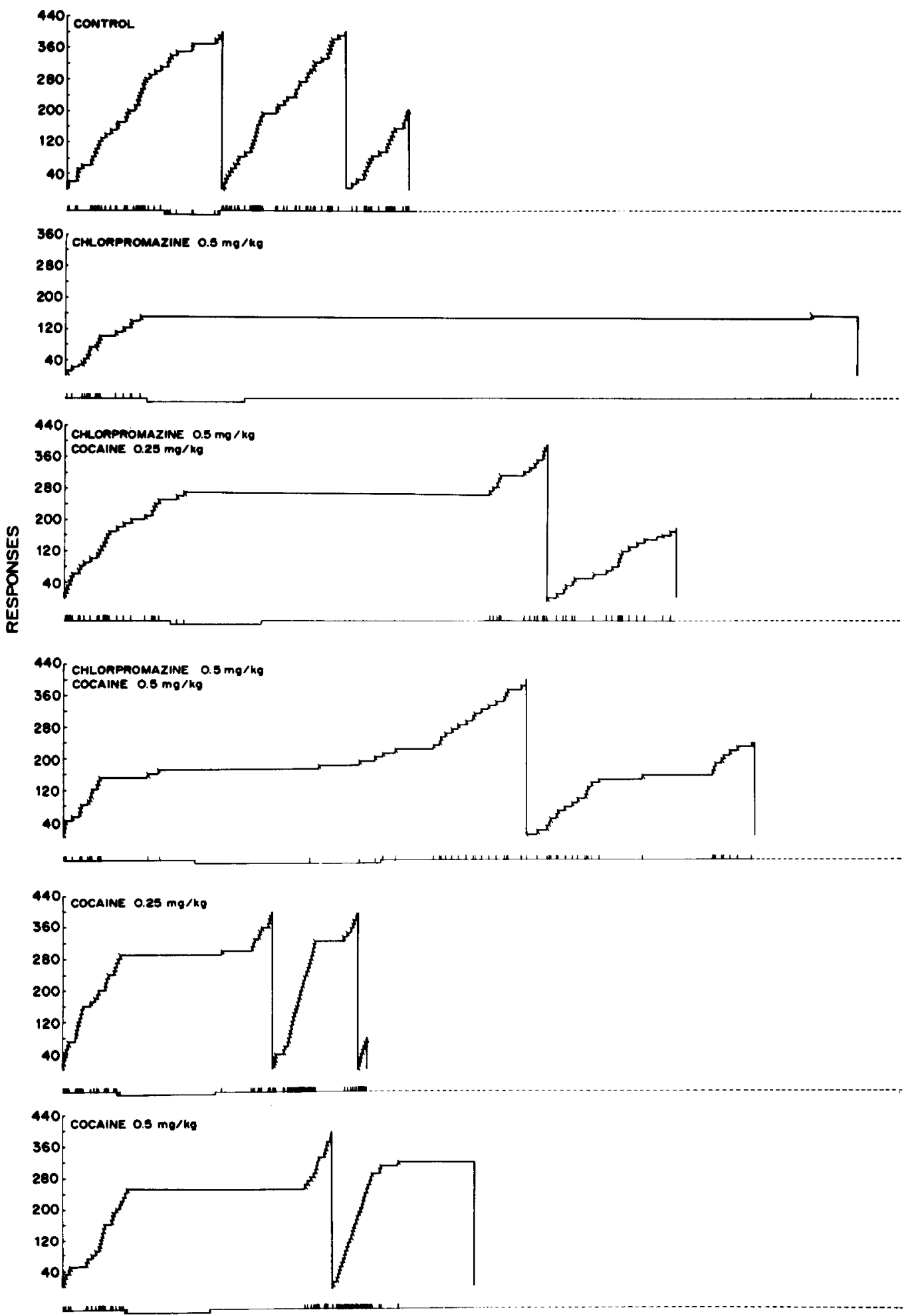

FIG. 8. Cumulative response records which illustrate the effects of chlorpromazine and cocaine individually and in combination on food reinforced behavior in 1 animal. Each vertical mark on the horizontal line represents presentation of 1 food pellet ie. 10 responses were emitted between successive vertical lines. Downward displacement of the horizontal line indicates onset of the cocaine or saline infusion and the horizontal length of this deflection indicates the duration of the infusions. The actual duration of all infusions was constant but since the recorder stopped during the $1 \mathrm{~min}$ timeout following each reinforcemont, the lengths of the negative deflections vary. The length of the deflection is inversely proportional to the amount of responding which occurred during the infusion. The end of the session is indicated by the change in character of the horizontal line from a solid to a broken form. 

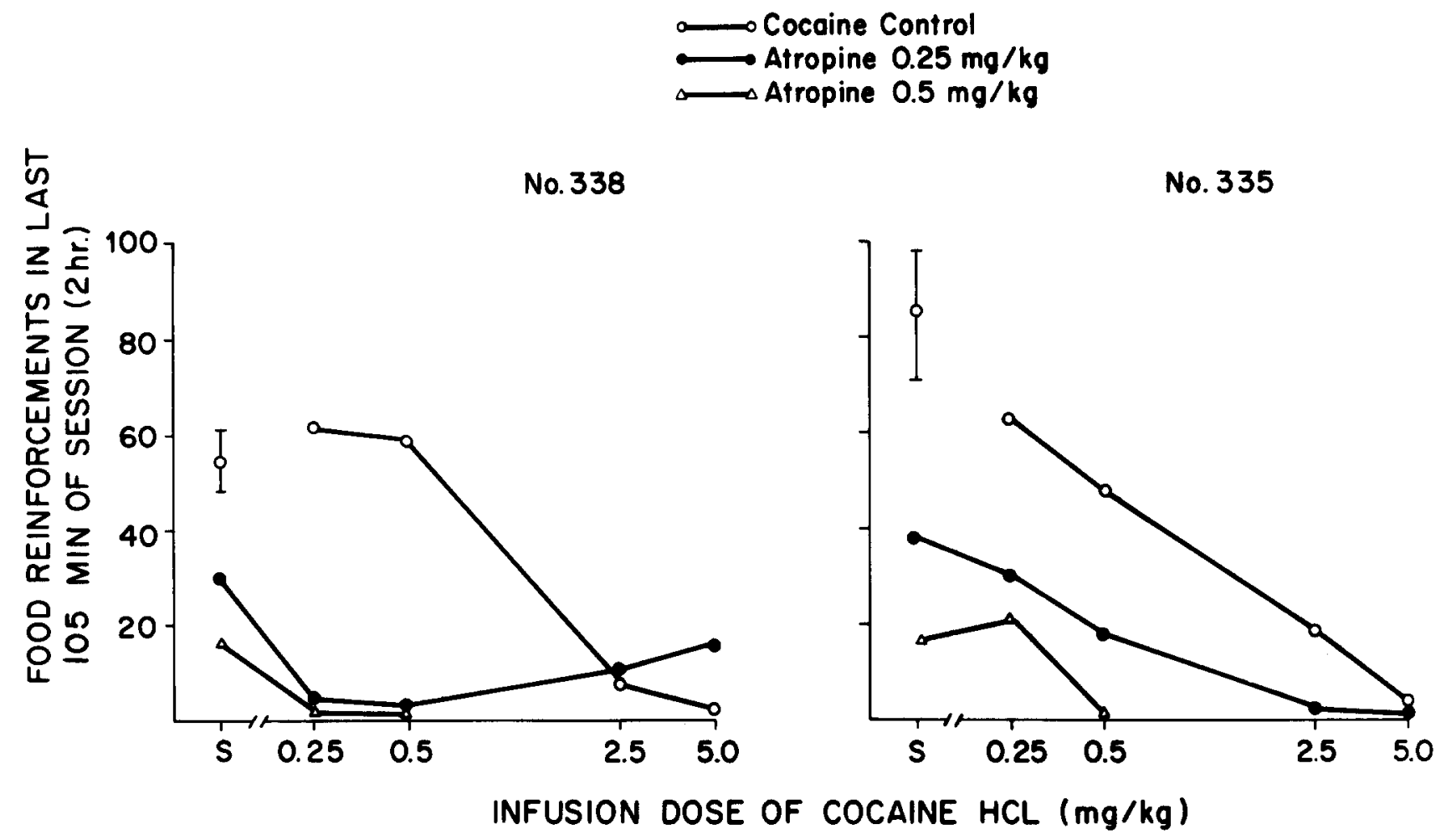

FIG. 9. Number of food reinforcements obtained during the last hour and $45 \mathrm{~min}$ of the $2 \mathrm{hr}$ session as a function of the pretreatment dosage of atropine and the infusion dosage of cocaine, individually and in combination. The brackets and superimposed symbol indicate the range and mean obtained from 5 control sessions i.e. animal received both a saline pretreatment and a saline infusion. The points above the letter $S$ indicate the effects seen with atropine pretreatment alone.

of a general behavioral antagonism between the two agents. Perhaps this may indicate that these dosages of atropine may increase the reinforcing efficacy of cocaine. The interaction of these agents on food reinforced behavior awaits further attempts at clarification as does that of cocaine and chlorpromazine. The fact that both atropine and chlorpromazine enhance cocaine self-administration; whereas their interaction with cocaine on food reinforced behavior is in the former case potentiation and in the latter case antagonism suggests that there is not a common mechanism for their effect on cocaine self-administration. These present results also dictate the complexity of analyzing the mecha- nisms involved in delineating the effects of atropine and chlorpromazine on cocaine based reinforcement and on those factors controlling cocaine self-administration.

\section{ACKNOWLEDGEMENT}

The authors wish to thank Mr. Robert Jackson for his technical assistance in completing this research project and to Mr. John Holbrook and Miss Judy Buelke for their assistance in the preparation of this manuscript. The authors also wish to thank Smith Kline and French Laboratories for the complimentary supply of chlorpromazine used in this research project. 
No. 335 FA-10 (FOOD PELLETS)
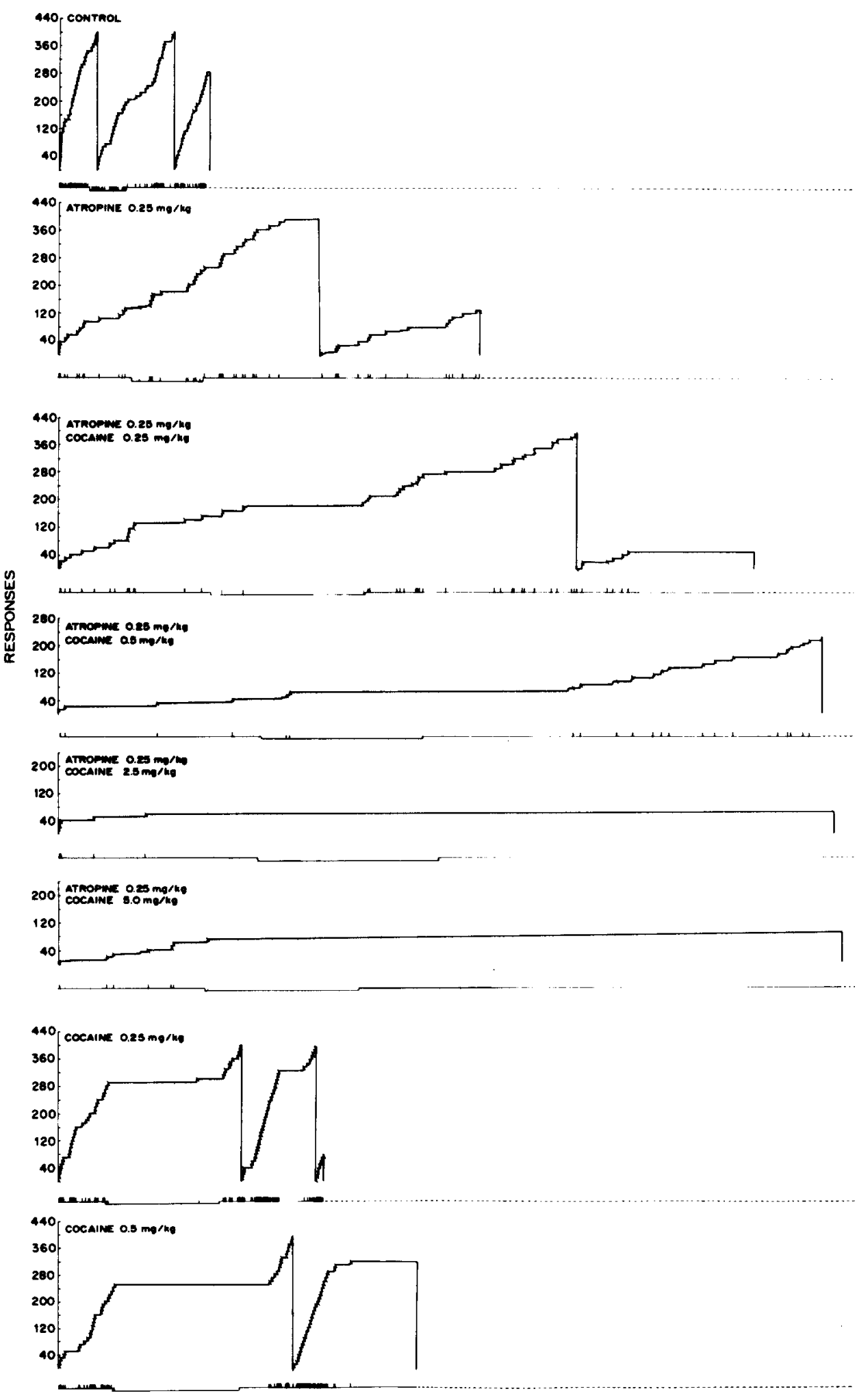

FIG. 10. Cumulative response records from 1 animal which illustrate the effects of atropine and cocaine individually and in combination on food reinforced behavior. Read as for Fig. 8. 


\section{REFERENCES}

1. Balster, R. L. and C. R. Schuster. Fixed-interval schedule of cocaine reinforcement: effect of dose and infusion duration. $J$. exp. Analysis Behav. 20: 119-129, 1973.

2. Brown, H. D-amphetamine-chlorpromazine antagonism in a food reinforced operant. J. exp. Analysis Behav. 6: 395-398, 1963.

3. Carlton, P. Augmentation of the behavioral effects of amphetamine by atropine. J. Pharmac. exp. Ther. 132: 91-96, 1961.

4. Deneau, G., T. Yanagita and M. H. Seevers. Self-administration of psychoactive substances by the monkey. Psychopharmacologia 16: 30-48, 1969.

5. Goldberg, S., F. Hoffmeister, U. Schlichting and W. Wuttke. A comparison of pentobarbital and cocaine self-administration in thesus monkeys: effects of dose and fixed-ratio parameter. $J$. Pharmac. exp. Ther. 179: 277-283, 1971.

6. Lu, T., J. L. Claghorn and J. C. Schoolar. Chronic administration of d-amphetamine and chlorpromazine in rats. Eur. $J$. Pharmac. 21: 61-65, 1973.

7. Pickens, R. and T. Thompson. Cocaine-reinforced behavior in rats: effect of reinforcement magnitude on fixed ratio size. $J$. Pharmac. exp. Ther. 161: 122-129, 1968.

8. Scheckel, C. and E. Boff. Behavioral effects of interacting imipramine and other drugs with d-amphetamine, cocaine, and tetrabenazine. Psychopharmacologia 5: 198-208, 1964.
9. Stein, L. New methods for evaluating stimulants and antidepressants. In: The First Hahnemann Symposium of Psychosomatic Medicine. Philadelphia: Lea and Febiger Inc., 1962, p. 297.

10. Whitehouse, J., A. Lloyd and S. A. Fifer. Comparative effects of atropine and methylatropine on maze acquisition and eating. J. comp. physiol. Psychol. 58: 475-476, 1964.

11. Wilson, M. C., M. Hitomi and C. R. Schuster. Psychomotor stimulant self-administration as a function of dosage per injection in the rhesus monkey. Psychopharmacologia 22: 271281,1971 .

12. Wilson, M. C. and C. R. Schuster. The effects of chlorpromazine on psychomotor stimulant self-administration in the rhesus monkey. Psychopharmacologia 26: 115-126, 1972.

13. Wilson, M. C. and C. R. Schuster. The effects of stimulants and depressants on cocaine self-administration behavior in the rhesus monkey. Psychopharmacologia 31: 291-304, 1973.

14. Wilson, M. C. and C. R. Schuster. Cholinergic influence on intravenous cocaine self-administration by rhesus monkeys. Pharmac. Biochem. Behav. 1: 643-649, 1973.

15. Woods, J. and C. R. Schuster. Reinforcement properties of morphine, cocaine, and SPA as a function of unit dose. Int. J. Addict. 3: 231-240, 1968. 